\title{
Overexpression of RNF2 is positively associated with ovarian carcinoma aggressiveness and indicative of poor patient survival
}

\author{
Xiao-Qing $\mathrm{Li}^{1, *}$, Wei-Peng He ${ }^{1, *}$, Wen-Hui Hou ${ }^{1}$, Jie-Wei Chen ${ }^{2}$, Rong-Rong Fan ${ }^{1}$, \\ Lin-Jing Yuan ${ }^{1}$, Gui-ping Yang ${ }^{1}$, Mu-Yan Cai ${ }^{2}$, Li Chen ${ }^{2}$, Jie Li ${ }^{1}$, Shan-Yang He ${ }^{1}$, Dan \\ $\mathrm{Xie}^{2}$, Guo-Fen Yang ${ }^{1}$, Ze-Shan You ${ }^{1}$ \\ ${ }^{1}$ Department of Gynecology, the First Affiliated Hospital, Sun Yat-Sen University, 510080 Guangzhou, China \\ ${ }^{2}$ State Key Laboratory of Oncology in South China, Cancer Center, Sun Yat-Sen University, 510060 Guangzhou, China \\ *These authors have contributed equally to this work \\ Correspondence to: Guo-Fen Yang, e-mail: pgf_yang@126.com \\ Ze-Shan You, e-mail: youzeshan888@21cn.com
}

Keywords: ovarian carcinoma, RNF2, immunohistochemistry, prognosis

Received: December 30, $2015 \quad$ Accepted: March 31, 2016

Published: April 25, 2016

ABSTRACT

It has been reported that RNF2, a core member of the polycomb repressor complex 1 (PRC1) complex, is frequently overexpressed in several types of human cancers. However, abnormalities in RNF2 in ovarian carcinoma and its potential clinical/ prognostic significance have not been elucidated. In this study, immunohistochemistry and fluorescence in situ hybridization were employed to examine the protein expression and amplification status of RNF2 in $\mathbf{3 0}$ normal ovaries, 30 ovarian cystadenomas, $\mathbf{4 0}$ borderline ovarian tumors and 170 ovarian carcinomas. Patient survival rate was evaluated using receiver-operator curve (ROC) analysis. Our result showed that overexpression of RNF2 was observed in none of the normal ovaries, in $7 \%$ of cystadenomas, in $15 \%$ of borderline tumors, and in $41 \%$ of ovarian carcinomas, respectively $(P<0.01)$. In addition, amplification of RNF2 was detected in $12.3 \%$ of ovarian carcinomas. Correlation analysis demonstrated that overexpression of RNF2 in ovarian carcinomas was positively associated with increased cell proliferation, ascending histological grade, later $\mathrm{pT} / \mathrm{pN} / \mathrm{pM}$ and/or more advanced FIGO stages $(P<0.05)$. In univariate survival analysis of the carcinoma cohorts, a significant association was observed between overexpression of RNF 2 and shortened patient survival (mean 47.4 months versus 97.0 months, $P<0.001$ ). Importantly, multivariate analysis demonstrated that RNF2 expression was a significant independent prognostic factor for overall survival of the carcinoma patients $(P=0.033)$. These findings provide evidence supporting that the overexpression of RNF2 might be a representation of a more aggressive cancer phenotype in ovarian carcinoma. Furthermore, RNF2 might serve as an independent biomarker indicative of poor prognosis in patients with ovarian carcinoma.

\section{INTRODUCTION}

Ovarian cancer is the most lethal gynecological malignancy that is responsible for over 140,000 deaths worldwide annually $[1,2]$. Unfortunately, most women with ovarian cancer were typically diagnosed at advanced stage (FIGO III/IV stage) due to the insidious, asymptomatic onset and the lack of effective methods for early detection. The 5-year survival rate of patients at advanced stage of ovarian cancer is $<30 \%$, while that of patients diagnosed at earlier stages (when the tumor is still confined to the ovary) is up to $90 \%[2,3]$. It is known that the most common malignant ovarian tumor is ovarian carcinoma. Similar to other types of human cancers, the pathogenesis of ovarian carcinoma is a multistep process, which involves a wide variety of abnormal genetic changes [4]. Better understandings of the molecular changes that are involved in the tumorigenesis and/or 
Table 1: The expression of RNF2 in normal ovaries and in benign and malignant epithelial ovarian tumors ${ }^{\mathrm{a}}$

\begin{tabular}{lccc}
\hline & & \multicolumn{2}{c}{ RNF2 protein } \\
\cline { 2 - 4 } & All cases & Normal expression & Overexpression \\
\hline Normal ovaries & 30 & $30(100 \%)$ & $0(0)$ \\
Cystadenomas & 30 & $28(93 \%)$ & $2(7 \%)$ \\
Borderline tumors & 40 & $34(85 \%)$ & $6(15 \%)$ \\
Invasive carcinomas & 170 & $100(59 \%)$ & $70(41 \%)$ \\
\hline
\end{tabular}

${ }^{a}$ Values are $\mathrm{n}(\%)$. A significant increasing frequency of intensive expression of RNF2 was observed in cystadenomas, in borderline tumors and in invasive carcinomas $(P<0.01$, Chi-Square Test for Trend)

progression of ovarian carcinoma are important for the development of effective therapies that could ultimately improve the overall survival of patients suffering from this disease.

RNF2, also known as Ring1B, was previously identified as a member of Bmil-interacting proteins, which comprise group II polycomb group (PcG) proteins $[5,6]$. Acting as transcriptional repressors, PcG proteins are often highly expressed in many types of human cancers [7]. PcG proteins contain at least two main biochemically and functionally distinct Polycomb core complexes: Polycomb repressive complex 2 and 1 (PRC2 and PRC1, respectively) [8]. RNF2 is one of the Polycomb repressive complex 1 (PRC1) core members. It plays the role of an E3 ligase and acts on histone $\mathrm{H} 2 \mathrm{~A}$ at lysine 119, which affects transcriptional activity [9-11]. Previous studies revealed that various E3 ligases regulate p53 activity through directing degradation or altering its transcriptional activity $[12,13]$. Furthermore, a previous study demonstrated that RNF2 negatively regulates p53 expression and promotes tumor development in selective cancer cell types [14]. In recent studies, overexpression of RNF2 was detected in ductal breast carcinoma and lymphomas, further indicating its involvement in tumor progression $[7,15]$. All of these findings imply that upregulation of RNF2 may promote tumorigenesis and metastasis, which suggests that RNF2 could be a new biomarker for poor prognosis and a new target for therapy in selective cancers.

Unfortunately, the molecular status of RNF2 in ovarian carcinoma, and its clinical and prognostic relevance have yet to be illuminated. In the present study, we examined the protein expression dynamics and amplification status of RNF2 by immunohistochemistry and FISH, respectively, in a large cohort of normal, pathological, non-neoplastic and neoplastic ovarian tissues. Using various statistical models, we were able to assess the clinical and prognostic significance of RNF2 expression in our ovarian carcinoma cohort. The results from our study provide important insights into the role of RNF2 in the development and treatment of ovarian carcinoma.

\section{RESULTS}

\section{RNF2 protein expression in ovarian tissues}

According to IHC staining in ovarian tissues, positive expression of RNF2 was primarily localized to the nuclei. The criteria for evaluating the status of RNF2 expression was according to the percentage of positive cells stained in each tissue section $(>10 \%$ epithelial cells positive for RNF2 staining was defined as overexpression). As expected, overexpression of RNF2 was detected in 70/170 (41\%) cases of ovarian carcinomas. Importantly, the increases in frequencies of RNF2 overexpression in normal ovarian epithelium (0), benign cystadenomas (7\%), borderline tumors $(15 \%)$, and finally, in invasive carcinomas $(41 \%)$ were statistical significant $(P<0.01$, Table 1). Representative IHC staining for RNF2 and Ki-67 expression is presented in Figure 1.

\section{Association of RNF2 protein expression with clinical pathological features in ovarian carcinoma patients}

We also examined the relationship between RNF2 overexpression in ovarian cancer carcinomas and patients' clinical pathological features in order to determine if there are any potential associations between the two. Our results revealed a statistically significant correlation between RNF2 expression levels and histological grade of tumor, $\mathrm{pT} / \mathrm{pN} / \mathrm{pM}$ status, and FIGO stage $(P<0.05$, Table 2). However, no significant correlation was observed between RNF2 expression and other clinical pathological features displayed by the ovarian carcinomas cohort, such as tumor histological type and the patient's age $(P>0.05$, Table 2). 


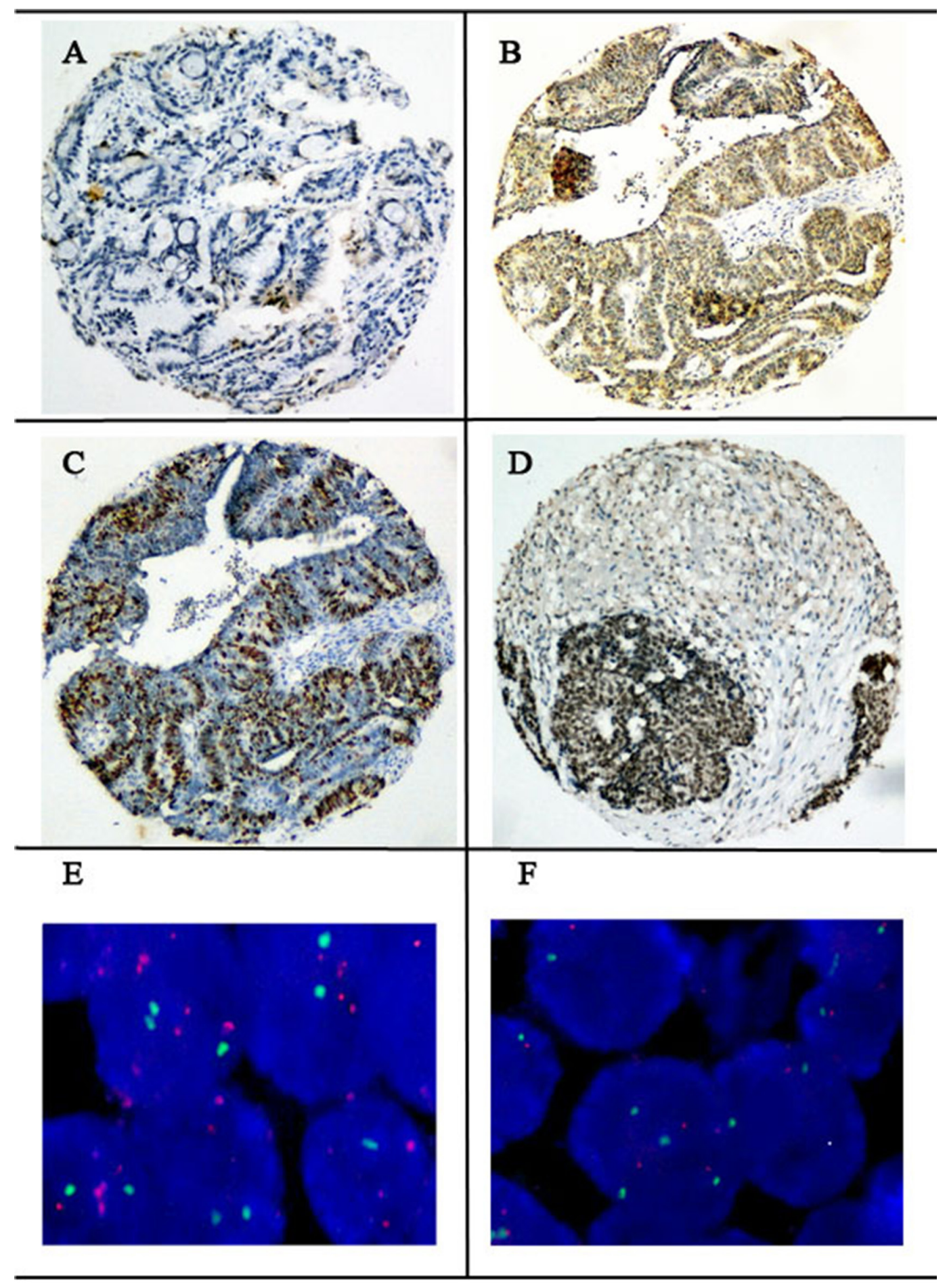

Figure 1: Immunohistochemical staining of RNF2 protein and Ki-67 in human ovarian carcinoma tissues. A. A case of primary ovarian carcinoma (case 11) demonstrated normal expression of RNF2 protein, in which less than 10\% of tumor cells showed high immunoreactivity of RNF2 protein in the nuclei (Original magnifications, $\times 100$ ). B. Overexpression of RNF2 protein was observed in a case of primary ovarian carcinoma (case 145), in which more than $80 \%$ tumor cells revealed positive immunostaining of RNF2 in the nuclei (Original magnifications, $\times 100$ ). C. Positive expression of Ki-67 (Original magnifications, $\times 100$ ) was observed in more than $60 \%$ of carcinoma cells. D. Another case of primary ovarian carcinoma (case 33) had overexpression of RNF2, in which more than $90 \%$ tumor cells showed positive nuclear immunostaining of RNF2 (Original magnifications, $\times 100$ ). E. Amplification of the RNF2 gene was observed by FISH in the same ovarian carcinoma case (case 33), in which RNF2 gene signals (red) were detected at least 3 times stronger than the signals for chromosome 1 centromere (green). (Original magnifications, $\times 1000$ ). F. A normal ovarian tissue showed normal copy numbers of RNF2 gene by FISH, in which RNF2 gene signals (red) were detected about 1 times as many gene signals than centromere signals of chromosome 1 (Original magnifications, $\times 1000)$. 
Table 2: Association of RNF2 expression with patient's clinico-pathological features in ovarian carcinomas

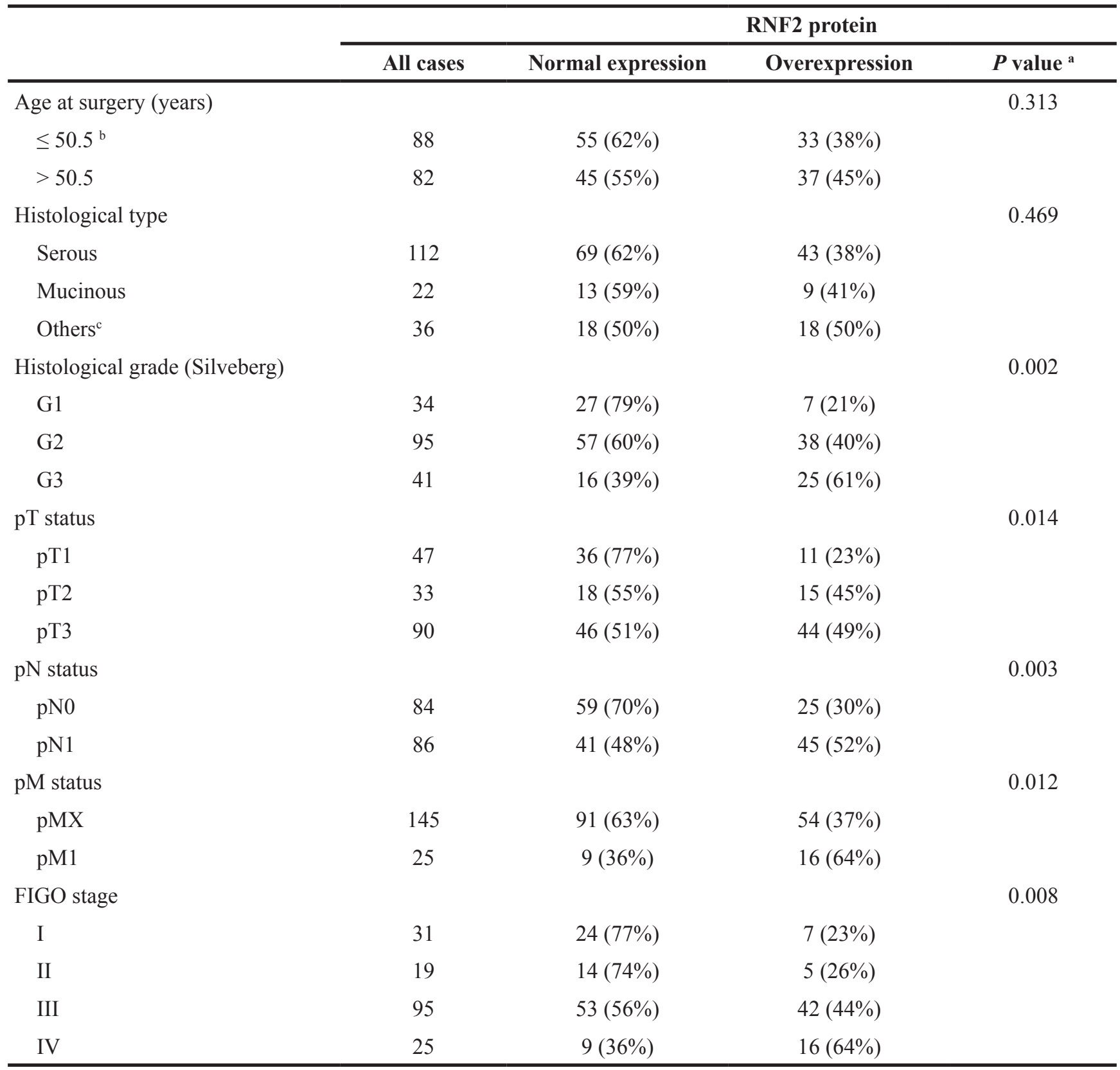

${ }^{\mathrm{a}}$ Chi-square test

${ }^{\mathrm{b}}$ Mean age

'Endometrioid, Clear cell and Undifferentiated types

\section{Relationship between clinical pathological variables, RNF2 expression, and ovarian carcinoma patient survival as determined by univariate survival analysis}

Cumulative survival curves were calculated according to the Kaplan-Meier method in our univariate survival analyses, Differences in survival time were assessed using the log-rank test. In order to confirm the representativeness of the ovarian carcinoma patient population in our cohorts, we analyzed established prognostic predictors of patient survival. Our analyses demonstrated that some well-known clinical pathological prognostic parameters, such as tumor histological grade $(P=0.008)$, pT status $(P=0.001)$, pN status $(P<0.001)$, pM status $(P<0.001)$, and FIGO stage $(P<0.001)$ showed statistically significant impact on patient survival (Table 3). With respect to RNF2 expression, we demonstrated that overexpression of RNF2 was positively correlated with adverse overall survival in patients with ovarian carcinoma $(P<0.001$, Table 3, Figure 2). 
Table 3: Clinical pathological parameters and expression of RNF2 for prognosis of 170 patients with ovarian carcinoma by univariate survival analysis (log-rank test)

\begin{tabular}{|c|c|c|c|c|}
\hline Variable & All cases & $\begin{array}{l}\text { Mean survival } \\
\text { (months) }\end{array}$ & $\begin{array}{l}\text { Median survival } \\
\text { (months) }\end{array}$ & $P$ value \\
\hline Age at surgery (years) & & & & 0.359 \\
\hline$\leq 50.7^{\mathrm{a}}$ & 88 & 82.2 & 136.0 & \\
\hline$>50.7$ & 82 & 77.7 & 55.0 & \\
\hline Histological type & & & & 0.338 \\
\hline Serous & 112 & 71.6 & 55.0 & \\
\hline Mucinous & 22 & 72.2 & $\mathrm{NR}^{\mathrm{b}}$ & \\
\hline Others & 36 & 113.9 & NR & \\
\hline Histological grade (Silveberg) & & & & 0.008 \\
\hline G1 & 34 & 100.3 & 136.0 & \\
\hline G2 & 95 & 83.8 & 62.0 & \\
\hline G3 & 41 & 46.0 & 28.0 & \\
\hline pT status & & & & 0.001 \\
\hline pT1 & 47 & 98.6 & NR & \\
\hline pT2 & 33 & 78.3 & 66.0 & \\
\hline pT3 & 90 & 64.3 & 34.0 & \\
\hline $\mathrm{pN}$ status & & & & $<0.001$ \\
\hline pNo & 84 & 96.0 & 136.0 & \\
\hline $\mathrm{pN} 1$ & 86 & 52.4 & 28.0 & \\
\hline pM status & & & & $<0.001$ \\
\hline $\mathrm{pMX}$ & 145 & 90.9 & 136.0 & \\
\hline pM1 & 25 & 22.2 & 13.0 & \\
\hline FIGO stage & & & & $<0.001$ \\
\hline I & 31 & 116.5 & NR & \\
\hline II & 19 & 112.2 & NR & \\
\hline III & 95 & 70.2 & 37.0 & \\
\hline IV & 25 & 22.2 & 13.0 & \\
\hline RNF2 expression & & & & $<0.001$ \\
\hline Normal expression & 100 & 97.0 & 136.0 & \\
\hline Overexpression & 70 & 47.4 & 28.0 & \\
\hline
\end{tabular}

${ }^{a}$ Mean age

${ }^{b}$ Not reached

Independent prognostic factors of ovarian carcinoma as determined by multivariate Cox regression analysis

The variables observed to have prognostic impact in our univariate analysis might also be covariates. In other words, the expression of RNF2 protein, as well as other clinical pathological parameters, such as tumor histological grade, $\mathrm{pT} / \mathrm{pN} / \mathrm{pM}$ status, and FIGO stage, might also significantly impact patient survival in multivariate analysis. We found that the expression of RNF2 was an independent prognostic factor for adverse 
overall survival (relative risk: 1.716, CI: 1.046-2.818, $P=0.033)$, as well as $\mathrm{pN}$ status $(P=0.021)$ and FIGO stage $(P<0.001)$ (Table 4).

\section{Relationship between clinical pathological parameters, RNF2 protein expression, and ovarian carcinoma patient survival as determined by ROC analysis}

ROC analysis for each clinical pathological parameters and the expression of RNF2 protein (area under the curve $[\mathrm{AUC}]=0.629, P=0.004$ ) were performed in order to assess patient's survival status (Figure 3). The ROC for each clinical pathological parameter was fitted to the curve closest $(0.0,1.0)$ to maximize both specificity and sensitivity of the result.

\section{Amplification of RNF2 in ovarian tumor TMA}

FISH analysis was informative in 106/170 (62.4\%) of ovarian carcinomas, 22/40 of borderline ovarian tumors and $14 / 30$ of ovarian cystadenomas in this study. Samples showing FISH signal and samples displaying weak target signals or those with a strong background were categorized as non-informative cases. FISH results suggested that amplification of RNF2 was detected in none of the ovarian cystadenomas and borderline tumor tissues; but was detected in $12.3 \%(13 / 106)$ of the informative ovarian carcinomas. Overexpression of RNF2 was observed in each of the 13 cases that displayed RNF2 amplification (Figure 1D). Among the remaining 93 informative cancers that showed no RNF2 amplification, 62 (66.7\%) cases displayed normal expression of RNF2, while the other 31 (33.3\%) cases showed overexpression of RNF2.

\section{Correlation between RNF2 expression and cell proliferation in ovarian carcinoma}

We examined the expression of Ki-67 by IHC in our cohort-TMAs to evaluate the relationship between RNF2 expression in ovarian carcinoma and cell proliferation. RNF2 and Ki-67 expressions were detected in 155 of the 170 samples. Among the 65 cases that displayed overexpression of RNF2, an average

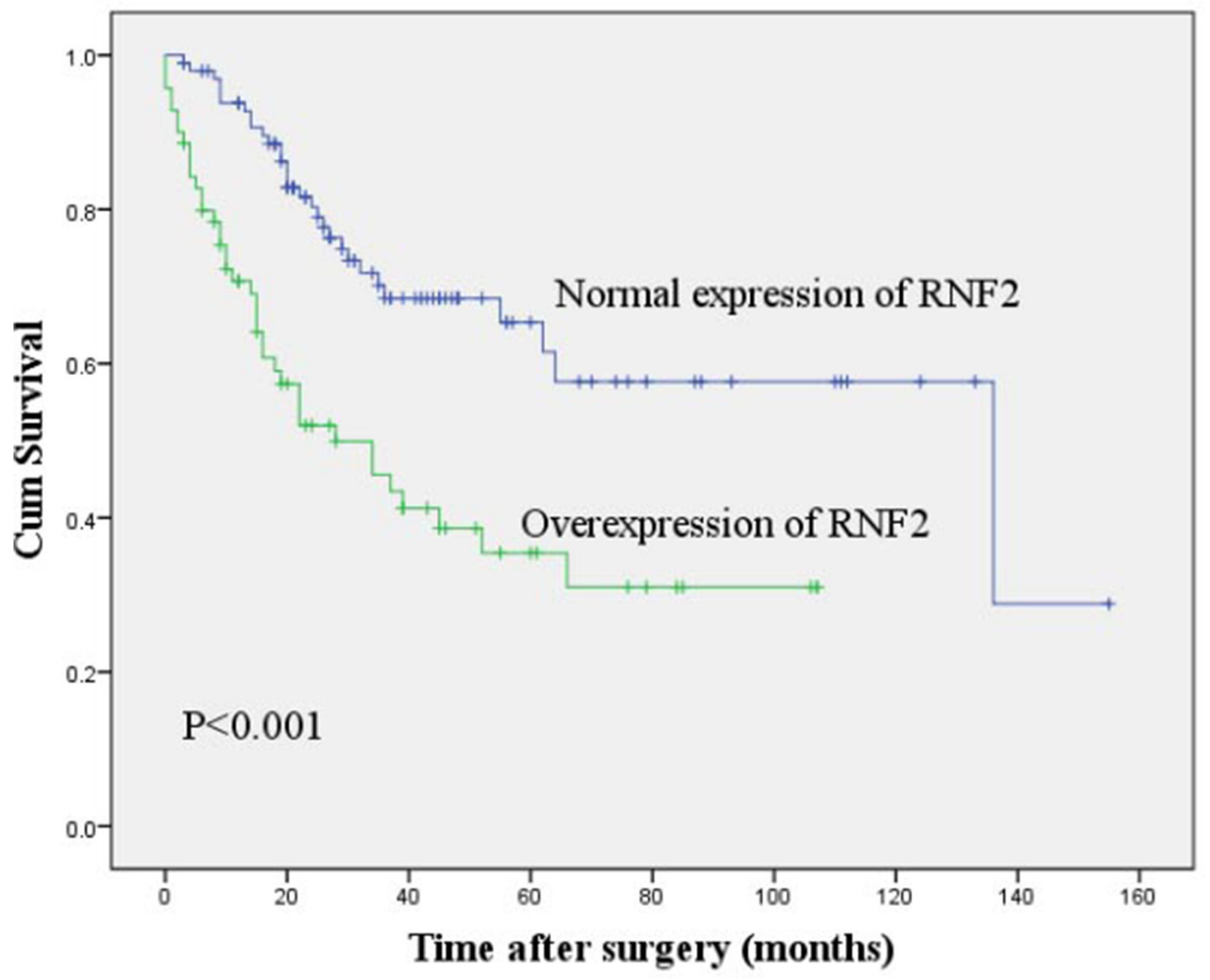

Figure 2: Kaplan-Meier survival analysis of RNF2 expression in 170 patients with invasive ovarian carcinoma (logrank test). Probability of patient survival: normal expression of RNF2, $n=100$; overexpression of RNF2, $n=70(p<0.001)$. 
Table 4: Multivariate analysis on overall survival (Cox regression model)

\begin{tabular}{lccc}
\hline Variable & Relative risk & 95\% Confidence interval & P value \\
\hline RNF2 $^{\mathrm{a}}$ & 1.716 & $1.046-2.818$ & 0.033 \\
pN status $^{\mathrm{b}}$ & 1.840 & $1.097-3.086$ & 0.021 \\
FIGO stage $^{\mathrm{c}}$ & 3.297 & $2.211-4.916$ & 0.000 \\
\hline
\end{tabular}

${ }^{\mathrm{a} O v e r e x p r e s s i n} v s$ Normal expression

${ }^{\mathrm{b}} \mathrm{pN} 1$ vs $\mathrm{pN} 0$

'Stage IV vs Stage III $v s$ Stage II vs Stage I

of $31.5 \%$ of the ovarian carcinoma cells also stained positive for Ki-67. However, the percentage of Ki-67positive cells in the remaining 90 cases that exhibited normal expression of RNF2 was only $19.7 \%(P<0.001$, Figure 4).

\section{DISCUSSION}

The incidence of ovarian carcinoma in developing countries, such as China and Singapore, has been on the rise in recent years [2]. Despite that enormous efforts have been made to develop surgical and chemotherapeutic approaches for the treatment of ovarian carcinoma, little improvement in patient outcomes associated with ovarian carcinoma has been achieved over the past decade. The main contributing factors for the adverse outcomes of ovarian carcinoma include delayed detection and diagnosis and lack of effective target drugs for anticancer therapy. Therefore, it is important to identify novel molecular abnormalities in ovarian carcinomas that could be used as therapeutic targets for effective treatment of the disease, which would ultimately lead to improvement in overall survival among patients with ovarian carcinoma.

As one of the most common chromosomal aberrations in cancers, genomic alterations or epigenetic changes in human chromosome 1q have been identified in several types of cancers, such as lung cancer, breast cancer, colorectal cancer, hepatocellular cancer and urothelial carcinoma [16-20]. These observations imply that human chromosome 1q might contain oncogenes that are related to tumorigenesis and/or progression of different types of cancer. Recent studies also showed that PcG proteins are highly expressed in various kinds of human cancer and could function as transcriptional repressors. In addition, others have shown that RNF2, a core member of the PRC1 complex, is encoded by chromosome 1q25.3 and is an oncogenic and pro-metastatic factor in Melanoma [10]. To further support the potential role of RNF2, overexpression of RNF2 was demonstrated in selective cancers [7, 15]. Furthermore, knockdown of RNF2 in
HeLa cells led to morphological changes and inhibition of cell proliferation [21]. However, the expression dynamics of RNF2 in ovarian carcinomas has yet to be illuminated, and its clinical pathological and/or prognostic values in ovarian carcinomas remain unclear. In this study, we investigated the molecular status of RNF2 and its clinical pathological significance and survival predictive value in ovarian carcinomas.

We first examined the protein expression of RNF2 by IHC in a large cohort of ovarian tissues, including a group of carcinoma tissues supplemented with complete patients' clinical pathological and follow-up data. The results demonstrated that the expression level of RNF2 protein among all normal ovarian specimens was low or absent. However, in our ovarian tumor specimens, there was a significant gradual increase in rate of overexpression of RNF2 starting from benign adenoma to borderline tumor and finally, to carcinoma. These results strongly implicated that overexpression of RNF2 might be involved in tumorigenesis of ovarian carcinoma. In addition, we found that overexpression of RNF2 in our ovarian carcinoma cohorts was strongly associated with an ascending histological grade and clinical stage (pT/ $\mathrm{pN} / \mathrm{pM}$ and FIGO stage) of the tumor. These findings suggested that up-regulated expression of RNF2 might represent an acquired malignant phenotypic feature of tumor cells and might play an important role in the development and/or progression of ovarian carcinoma. Furthermore, Kaplan-Meier curves and multivariate Cox proportional hazard regression analysis indicated that overexpression of RNF2 was a strong and independent predictor for poor overall survival in patients with ovarian carcinoma. Taken together, these findings suggested that RNF2 expression is a reliable biomarker for prognosis of ovarian cancer.

It has been well established that gene amplification is a common pathological mechanism of oncogene overexpression in human cancers [22]. In order to determine whether gene amplification leads to the overexpression of RNF2 in ovarian carcinomas, the amplification status of RNF2 was examined by 


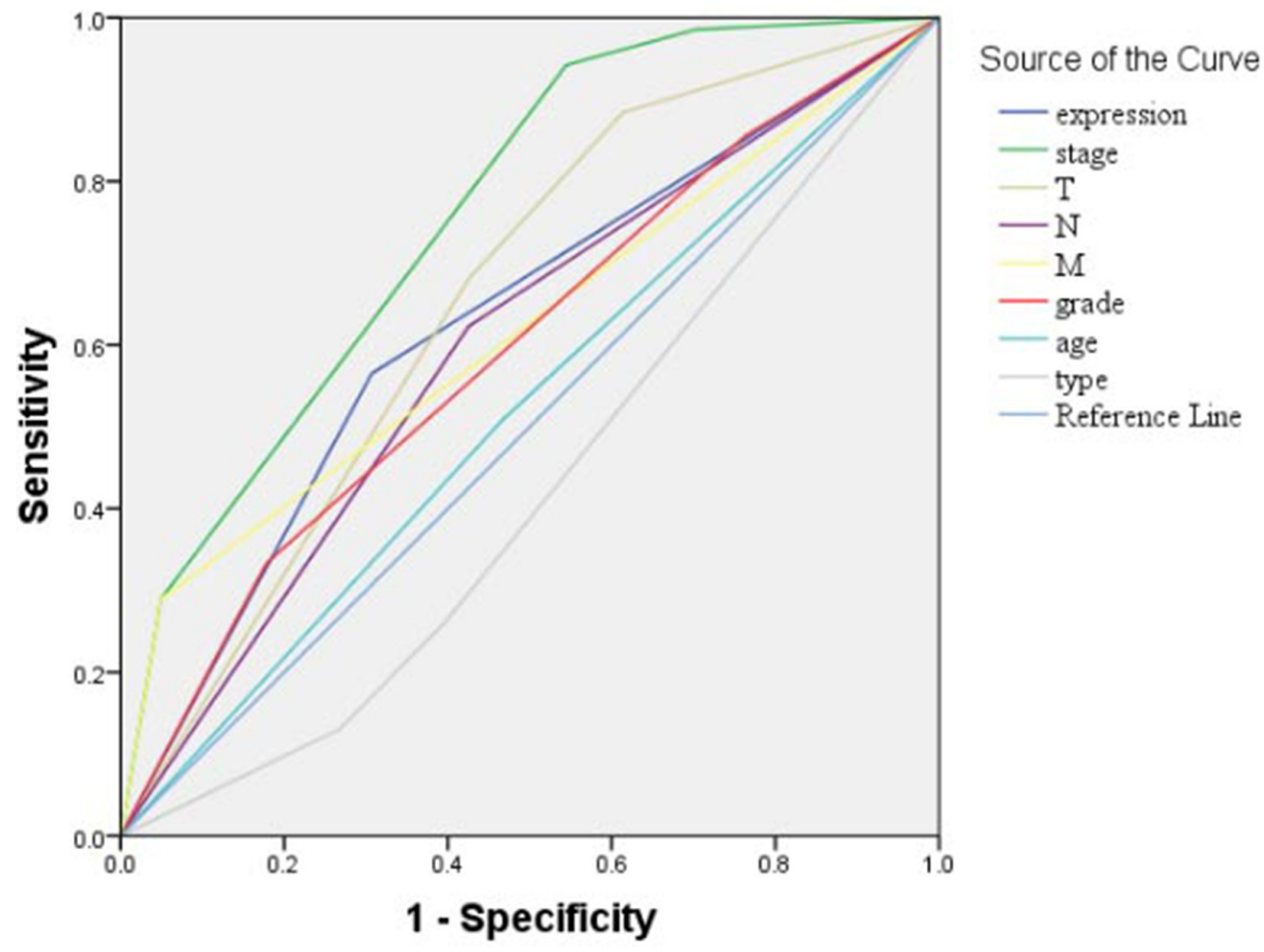

Figure 3: ROC curve analysis on different clinical pathological parameters and RNF2 protein expression was performed to evaluate survival status. FIGO stage (area under the curve $[\mathrm{AUC}]=0.760, P<0.001$ ), RNF2 protein expression ([AUC] $=0.629$, $P=0.004), \mathrm{pT}$ ([AUC] $=0.656, P=0.001), \mathrm{pN}([\mathrm{AUC}]=0.599, P=0.029), \mathrm{pM}([\mathrm{AUC}]=0.620, P=0.008)$, Pathological grading $([\mathrm{AUC}]=0.597$, $P=0.032)$ is significantly associated with survival.

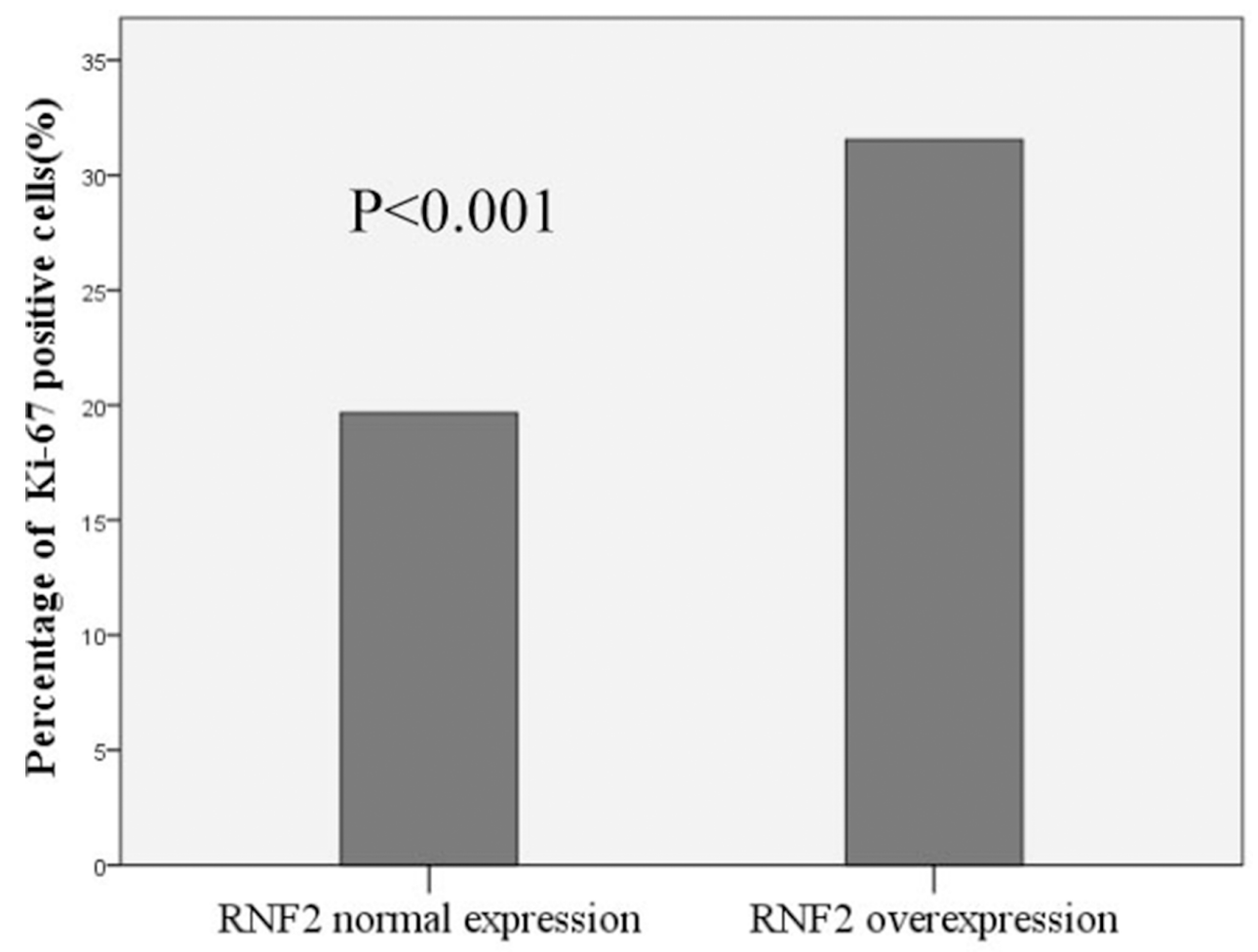

Figure 4: The relationship between RNF2 expression and Ki-67 in ovarian carcinoma. The average percentage of ovarian cancer cells stained positive with Ki-67 antibody in RNF2 normal expression group (19.7\%), which is significantly lower than that in RNF2 overexpression group $(31.5 \%, P<0.001)$. 
FISH. Among the 106 informative ovarian carcinoma cases that were detected by both IHC and FISH simultaneously, overexpression of RNF2 was detected in all (13/13) ovarian carcinomas that displayed RNF2 amplification. However, amplification of RNF2 was not observed in the other 31 ovarian carcinomas with RNF2 protein overexpression. These findings suggested that the expression level of RNF2 protein in ovarian carcinoma was associated, but not always coincided with RNF2 gene amplification. These results also implicated that the up-regulated protein expression of RNF2 in ovarian cancers is complex phenomenon that might be regulated by molecular mechanisms including transcriptional regulation and post-translational regulation in addition to gene amplification. Clearly, further investigations are needed to better understand the molecular mechanisms of up-regulated expression of RNF2 in ovarian carcinoma, as well as in other human cancers.

To date, the precise mechanism by which RNF2 exerts its effects on the tumorigenesis and/or progression of human malignant tumors remains elusive. Previous study observed that RNF2 acts as a ubiquitin E3 ligase, a negative regulators for $\mathrm{p} 53$ that promotes $\mathrm{p} 53$ degradation in selective cell lines, thereby, contributing to cell proliferation [14]. Conversely, knockdown of RNF2 induces apoptosis, which is at least partially dependent on p53 [21]. Therefore, previous study proposed that RNF2 might promote tumor development partially by negatively regulating p53 expression [14]. In melanoma, it has been found that RNF2 regulates different biological processes in the genesis and progression of melanoma by distinct molecular mechanisms [10]. Our current study has also illuminated some of the functions of the RNF2 protein. In our ovarian carcinoma cohort, we observed that there was a significant positive association between the level of RNF2 protein and Ki-67, which is an established marker crucial for cell proliferation. Based on these results, we speculated that RNF2 might potentially play an important role in the regulation of ovarian carcinoma cell proliferation, an activity that might be partially responsible for tumorigenesis and/or progression of the cancer. Further studies are essential to precisely illustrate the mechanism of RNF2 overexpression in the development and progression of ovarian carcinoma and other human tumors.

In summary, we demonstrated, for the first time, the protein expression and amplification patterns of RNF2 in a large cohort of human ovarian benign adenomas, borderline tumors, and malignant carcinomas, Our results suggested that overexpression of RNF2 not only plays an important role in the pathogenesis of ovarian carcinoma, but it might also serve as a useful prognostic molecular marker indicative of poor outcome in individual patient.

\section{PATIENTS AND METHODS}

\section{Patients and tissue specimens}

In this study, a total of 240 epithelial ovarian tumors (including 30 cases of benign, 40 cases of borderline and 170 cases of carcinomas) were obtained from the archives of paraffin-embedded tissues between 2000 and 2012 at the Department of Pathology, Cancer Center and the First Affiliated Hospital, Sun Yat-Sen University, Guangzhou, China. Data on survival time and clinical pathological parameters were also collected. All patients included in this study provided informed consent. Patients who had received complete resection and not received preoperative chemo-radiation therapy and had complete clinical followup data available for review were selected for this study. Patients who had incomplete clinical follow-up data or had died from unknown causes and emergencies were excluded. The mean age of the patients in our ovarian carcinoma cohort was 50.5 years, with a range of 2086 years. The clinical pathological parameters for these patients are summarized in Table 2. The use of these clinical materials for research purposes was reviewed and approved by the Medical Ethical Committee of Sun Yatsen University.

\section{Construction of tissue microarrays (TMA)}

The tissue microarray was constructed according to previously described methods [23]. Briefly, individual donor tissue block and matching histological H\&E stained slides were overlaid for tissue TMA sampling. The slides were reviewed by a senior pathologist, who marked representative areas of usable tumor tissues. The tissues were sampled with a tissue-arraying instrument (Beecher Instruments, Silver Spring, MD); 0.6-mmdiameter cylinder of tissue were selected in triplicates, removed from tumor areas of individual donor tissue block and re-embedded into a recipient paraffin block at the predetermined position. Subsequently, multiple sections (5- $\mu \mathrm{m}$ thick) were cut from the TMA block and mounted on microscopic slides.

\section{Immunohistochemistry (IHC)}

IHC studies were performed following a standard streptavidin-biotin-peroxidase complex method [23]. In brief, the constructed ovarian tissue microarrays were deparaffinized in xylene and rehydrated in a graded series of ethanol. Then, the endogenous peroxidase activity was blocked by $0.3 \%$ hydrogen peroxide for $20 \mathrm{~min}$. For antigen retrieval, tissue slides were heated in $10 \mathrm{mM}$ citrate buffer ( $\mathrm{pH}$ 6.0) in a pressure cooker for $10 \mathrm{~min}$. Subsequently, slides were incubated in $10 \%$ normal rabbit serum for $20 \mathrm{~min}$ to block nonspecific binding of the immunological reagents. The slides were then incubated 
with anti-RNF2 (Abcam, Cambridge, MA, diluted 1:100 in PBS) and anti-Ki-67 (Dako, Glostrup, Denmark, diluted 1:100 in PBS) overnight at $4^{\circ} \mathrm{C}$ in a moist chamber. After incubation with biotinylated rabbit anti-mouse immunoglobulin at a concentration of 1:100 for $30 \mathrm{~min}$ at $37^{\circ} \mathrm{C}$, the slides were reacted with a streptavidin-peroxdase conjugate for $30 \mathrm{~min}$ at $37^{\circ} \mathrm{C} .3^{\prime}-3^{\prime}$ diaminobenzidine was used as chromogen substrate, and nuclear counterstaining was performed with Mayer's hematoxylin. IHC positive RNF2 staining slides of breast cancer tissues were used as positive control, and a negative control was obtained by replacing the primary antibody with normal rabbit IgG.

Positive expression of RNF2 by IHC in ovarian cells was primarily confined in the nuclei (Figure 1B). Malignant and non-malignant tissues were scored for RNF2 and Ki-67 expression by assessing the sites of positive staining in the nuclei. The percentage of RNF2 and $\mathrm{Ki}-67$ positive cells in each tumor was calculated to assess the status of RNF2 and Ki-67 nuclear expression. A minimum of 500 tumor cells and 300 normal epithelial cells were counted in each tumor and in normal control. All histological evaluations were performed in a doubleblind manner by two independent expert pathologists (Dr. Chen JW and Xie D).

\section{Fluorescence in situ hybridization (FISH)}

Two-color FISH was performed using a Spectrum Red-labeled BAC clone (CH17-111I15) containing a RNF2 gene and a chromosome 1 centromere probe, which was labeled with Spectrum Green (Vysis, Downers Grove, IL) as an internal control. The FISH reaction was carried out as previously described with slight modifications [24]. Briefly, the deparaffinized ovarian carcinoma tissue sections were treated with proteinase $\mathrm{K}(400 \mu \mathrm{g} /$ $\mathrm{ml}$ ) at $37^{\circ} \mathrm{C}$ for $45 \mathrm{~min}$, followed by denaturing in $70 \%$ formamide, $2 \times \mathrm{SSC}$ at $75^{\circ} \mathrm{C}$ for $8 \mathrm{~min}$. Fifty nanograms of each probe were mixed in a $20 \mu$ hybridization mixture (containing 55\% formamide, $2 \times \mathrm{SSC}$, and $2 \mu \mathrm{g}$ human Cot1 DNA), denatured at $75^{\circ} \mathrm{C}$ for 6 minutes and then hybridized to the denatured tissue sections at $37^{\circ} \mathrm{C}$ for 24 hours. The slides were counterstained with $1 \mu \mathrm{g} / \mathrm{ml}$ DAPI in an anti-fade solution and were examined with a Zeiss Axiophot microscope equipped with a triple-band pass filter. FISH signals from a minimum of 300 tumor cells were evaluated per specimen. Gene amplification of RNF2 was defined as presence of either 6 (or more) RNF2 gene signals or more than 3 times as many gene signals than reference signals in tumor cells (Figure 1E).

\section{Statistical analyses}

Statistical analysis was carried out with the SPSS software (SPSS Standard version 20.0, SPSS Inc.). Chi-square tests were used to assess the association of RNF2 protein expression with the clinical pathological features of ovarian carcinoma patients. An independent sample t-test was performed to assess the expression of Ki-67 between groups with different levels of RNF2 expression. For univariate survival analysis, we obtained and assessed survival curves using the Kaplan-Meier method and compared different survival curves with the log rank test. Multivariate Cox regression analysis was performed on all parameters that were found to be significant in the univariate analysis. Differences between the clinical pathological features and estimation of the survival predictions were assessed by receiver operating characteristic curve (ROC) analysis. P value of $<0.05$ was considered to be statistically significant.

\section{CONFLICTS OF INTEREST}

The authors declare that there are no conflicts of interest.

\section{GRANT SUPPORT}

This study was supported by grants from the Nature Science Foundation of China (No. 81272855), the Nature Science Foundation of Guangdong Province (No. S2012010008810 and No. S2013010015403).

\section{REFERENCES}

1. Brian S. Gloss, Goli Samimi. Epigenetic biomarkers in epithelial ovarian cancer. Cancer Letters. 2014; 342:257-263.

2. Jemal A, Bray F, Center MM, Ferlay J, Ward E, Forman D. Global cancer statistics. CA Cancer J Clin. 2011; 61:69-90.

3. Jill S. Barnholtz-Sloan, Ann G. Schwartz, Faisal Qureshi, Suzanne Jacques, John Malone, Adnan R. Munkarah. Ovarian cancer: changes in patterns at diagnosis and relative survival over the last three decades. Am J Obstet Gynecol. 2003; 189:1120-1127.

4. DiSaia PJ, Bloss JD. Treatment of ovarian cancer: new strategies. Gynecol Oncol. 2003; 90:S24-S32.

5. Gretel Buchwald, Petra van der Stoop, Oliver Weichenrieder, Anastassis Perrakis, Maarten van Lohuizen, Titia K Sixma. Structure and E3-ligase activity of the RingRing complex of Polycomb proteins Bmil and Ring1b. The EMBO Journal. 2006; 25:2465-2474.

6. D Choi, S-J Lee, S Hong, I-H Kim, S Kang. Prohibitin interacts with RNF2 and regulates E2F1 function via dual pathways. Oncogene. 2008; 27:1716-1725.

7. Almudena Bosch, Konstantina Panoutsopoulou, Josep Maria Corominas, Ramón Gimeno, Gema Moreno-Bueno, Juan Martín-Caballero, Saleta Morales, Tania Lobato, Carles Martínez-Romero, Eduardo F. Farias, Xavier Mayol, Amparo Cano, Inmaculada Hernández-Muñoz. The Polycomb group protein RING1B is overexpressed in ductal 
breast carcinoma and is required to sustain FAK steady state levels in breast cancer epithelial cells. Oncotarget. 2014; 5:2065-2076. doi: 10.18632/oncotarget.1779.

8. Lund AH, van Lohuizen M. Polycomb complexes and silencing mechanisms. Curr Opin Cell Biol. 2004; 16: 239-246.

9. Petra van der Stoop, Erwin A. Boutsma, Danielle Hulsman, Sonja Noback, Mike Heimerikx, Ron M. Kerkhoven, J. Willem Voncken, Lodewyk F. A. Wessels, Maarten van Lohuizen. Ubiquitin E3 Ligase Ring1b/Rnf2 of Polycomb Repressive Complex 1 Contributes to Stable Maintenance of Mouse Embryonic Stem Cells. PLoS ONE. 2008; 3:e2235.

10. Rai K, Akdemir KC, Kwong LN, Fiziev P, Wu CJ, Keung EZ, Sharma S, Samant NS, Williams M, Axelrad JB, Shah A, Yang D, Grimm EA, Barton MC, Milton DR, Heffernan TP, Horner JW, Ekmekcioglu S, Lazar A, Ernst J, Chin L. Dual Roles of RNF2 in Melanoma Progression. Cancer Discov. 2015; 5:1314-1327.

11. Yingtao Zhang, Xin Li, Zhengming Chen, Gerold Bepler. Ubiquitination and Degradation of Ribonucleotide Reductase M1 by the Polycomb Group Proteins RNF2 and Bmi1 and Cellular Response to Gemcitabine. PLoS One. 2014; 9:e91186.

12. Li M, Brooks CL, Wu-baer F, Chen D, Baer R, Gu W. Mono-versus polyubiquitination: Differential control of p53 fate by Mdm2. Science. 2003; 302:1972-1975.

13. Jain AK, Barton MC. Making sense of ubiquitin ligases that regulate p53. Cancer Biol Ther. 2010; 10:665-672.

14. Su WJ, Fang JS, Cheng F, Liu C, Zhou F, Zhang J. RNF2/ Ring $1 \mathrm{~b}$ negatively regulates $\mathrm{p} 53$ expression in selective cancer cell types to promote tumor development. Proc Natl Acad Sci USA. 2013; 110: 1720-1725.

15. Margarita Sánchez-Beato, Esther Sánchez, Joaquín González-Carreró, Manuel Morente, Ana Díez, Lydia Sánchez-Verde, María Carmen Martín, Juan C Cigudosa, Miguel Vidal, Miguel A Piris. Variability in the expression of polycomb proteins in different normal and tumoral tissues. A pilot study using tissue microarrays. Mod Patho. 2006; 19:684-694.

16. Ma J, Gao M, Lu Y, Feng X, Zhang J, Lin D, Xiao T, Hu Z, Yuan J, Su K, Shipley J, Xue J, Gao Y. Gain of 1q25-32, 12q23-24.3, and 17q12-22 facilitates tumorigenesis and progression of human squamous cell lung cancer. J Pathol. 2006; 210: 205-213.

17. Mesquita B, Lopes P, Rodrigues A, Pereira D, Afonso M, Leal C, Henrique R, Lind GE, Jeronimo C, Lothe RA, Teixeira MR. Frequent copy number gains at 1q21 and 1q32 are associated with overexpression of the ETS transcription factors ETV3 and ELF3 in breast cancer irrespective of molecular subtypes. Breast Cancer Res Treat. 2013; 138: $37-45$.

18. Kassouf W, Agarwal PK, Herr HW, Munsell MF, Spiess PE, Brown GA, Pisters L, Grossman HB, Dinney CP, Kamat AM. Lymph node density is superior to TNM nodal status in predicting disease-specific survival after radical cystectomy for bladder cancer: analysis of pooled data from MDACC and MSKCC. J Clin Oncol. 2008; 26:121-126.

19. Ma NF, Hu L, Fung JM, Xie D, Zheng BJ, Chen L, Tang DJ, Fu L, Wu Z, Chen M, Fang Y, Guan XY. Isolation and characterization of a novel oncogene, amplified in liver cancer 1 , within a commonly amplified region at 1q21 in hepatocellular carcinoma. Hepatology. 2008; 47:503-510.

20. Poddighe PJ, Ramaekers FC, Smeets AW, Vooijs GP, Hopman AH. Structural chromosome 1 aberrations in transitional cell carcinoma of the bladder: interphase cytogenetics combining a centromeric, telomeric, and library DNA probe. Cancer Res. 1992; 52:4929-4934.

21. Wen W, Peng C, Kim MO, Ho Jeong C, Zhu F, Yao K, Zykova T, Ma W, Carper A, Langfald A, Bode AM, Dong Z. Knockdown of RNF2 induces apoptosis by regulating MDM2 and p53 stability. Oncogene. 2014; 33: 421-428.

22. Stark GR, Debatisse M, Giulotto E, Wahl GM. Recent progress in understanding mechanisms of mammalian DNA amplification. Cell 1989; 57: 901-908.

23. Yang GF, He WP, Cai MY, Li-Ru He, Jun-Hang Luo, Hai-Xia Deng, Xin-Yuan Guan, Mu-Sheng Zeng, Yi-Xin Zeng, Dan Xie. Intensive expression of Bmi-1 is a new independent predictor of poor outcome in patients with ovarian carcinoma. BMC Cancer 2010; 10:133.

24. Xie D, Sham JS, Zeng WF, Lin HL, Bi J, Che LH, Hu L, Zeng YX, Guan XY. Correlation of AIB1 overexpression with advanced clinical stage of human colorectal carcinoma. Hum Pathol 2005; 36: 777-783. 